\title{
Zur quantitativen Bestimmung des Wismuts durch Elektrolyse.
}

\author{
Von \\ KARI WIMMEnaUer.
}

\section{Einleitung.}

In den Handbüchern für die quantitative elektrolytische Bestimmung der Metalle ${ }^{1}$ finden sich von Vorschriften zur Bestimmung des Wismuts nur sehr wenige zur Anwendung empfohlen. Manche Autoren verzichten überhaupt auf die Angabe irgend welcher Methoden; so sagt Crasssen in der letzten Auflage seiner quantitativen Analyse durch Elektrolyse: „Wismut in dichter, metallischer Form quantitativ niederzuschlagen, ist bislang noch nicht gelungen. Es fällt aus allen Verbindungen mehr oder weniger schwammartig, so dals auf Wiedergabe der Vorschriften verschiedener Autoren verzichtet werden mufs."

Andere $^{2}$ entscheiden sich für die Vortmann'sche Amalgammethode ${ }^{3}$ nach welcher das Wismut zusammen mit Quecksilber als Amalgam ausgeschieden und gewogen wird. Dabei mufs das Quecksilber dem Wismut wenigstens um das Vierfache an Gewicht überlegen sein, wenn ein brauchbares Amalgam erhalten werden soll. Das Quecksilber wird als Oxyd oder als Chlorid gewogen, gelöst und der Wismutsalzlösung zugefügt. Nach beendeter Fällung wird die berechnete Menge des Quecksilbers vom Gesamtgewichte des Amalgams abgezogen. Die Differenz wird als Gewicht des gefundenen Wismuts angenommen.

1 Benutzt werden: A. Cuassen, quant. Analyse durch Elektrolyse, B. NEumans, Theorie und Praxis der Elektrolyse, Fr. Peters, angew. Elektrochemie, F. B. Anrens, Handbuch der Elektrochemie, und der Elektrochemikerkalender.

2 Vergl. Peters, angew. Elektroch. II, 2, S. 178 und Neumann, Theorie n. Praxis d. Elektrochem., S. 158.

${ }^{3}$ Ber. deutsch. chem. Ges. 24, 2759.

Z. anorg. Chem. XXVII. 
Es liegt anf der Hand, dafs diese indirekte Bestimmungsweise bedeutende Fehlerquellen enthalten mufs und den Hauptvorzug der elektrolytischen ror den anderen analytischen Methoden gar nicht zur Geltung kommen läfst, nämlich den, dafs sie gestatten, das Metall als solches zur Wägung zu bringen. Es wäre deshalb sehr wünschenswert, eine brauchbare direkte Bestimmungsmethode für das Wismut ausfindig zu machen.

Auf Veranlassung des Herrn Professor Dr. Medicus in Würzburg unterzog ich mich daher der Aufgabe, die wichtigsten von den in der Litteratur veröffentlichten Methoden zur direkten elektrolytischen Wismutbestimmung einer eingehenden Prüfung zu unterziehen und den Versuch zu machen, ob nicht doch durch geeignete Abänderungen der Versuchsbedingungen die Wismutelektrolyse einer allgemeineren Anwendung als bisher zugänglich gemacht werden könne.

Bei der Beurteilung der elektrolytischen Methoden ging ich von folgenden Gesichtspunkten aus:

1. Das Ausgangsmaterial für die Elektrolyse mufs in gut wägbarer und reiner Form vorhanden sein.

2. Die Lösung mufs ohne Umstände und Schwierigkeiten herzustellen sein.

3. Die Elektrolyse soll mit den allgemein üblichen Mitteln und Apparaten ausgeführt werden können.

4. Die Analyse soll in möglichst kurzer Zeit, längstens aber während eines Tages zu Ende geführt werden können.

5. Der Metallniederschlag soll ohne Verluste auswaschbar sein.

6. Die anzuwendenden Mengen des Wismuts sollen nicht zu gering sein und im allgemeinen nicht weniger als $0.1 \mathrm{~g}$ betragen.

Als Ausgangsmaterialien kommen eigentlich nur zwei Wismutverbindungen in Betracht, nämlich das Nitrat, $\mathrm{Bi}\left(\mathrm{NO}_{3}\right)_{3}, 5 \mathrm{H}_{2} \mathrm{O}$ mit $42.91 \%$ Bi und das Oxyd, $\mathrm{Bi}_{2} \mathrm{O}_{3}$ mit $89.65 \%$ Bi. Das Oxyd ist dem Nitrat vorzuziehen, weil es in sehr reiner und beständiger Form erhältlich ist. Man stellt es am besten durch Glühen des reinen basischen Nitrats dar.

Als Stromquellen benutzte ich ausschliefslich Akkumulatoren.

$\mathrm{Da}$ bei der Elektrolyse der Wismutsalze meist mit sehr geringen Stromstärken gearbeitet wird und die richtige Stromstärke sorgfältig eingehalten werden muls, so sind genaue Mel'sinstrumente erforderlich, welche das Ablesen von $0.01 \mathrm{Amp}$. noch gestatten. Ich 
benutzte Präzisionsinstrumente von Siemens \& HaLske und Normalinstrumente von HaRTMANN \& BRAUN.

Als Einschaltwiderstände eignen sich der schwachen Ströme wegen die sonst gebräuchlichen Drahtrheostaten nicht. Am zweckmälsigsten habe ich einen einfachen Wasserwiderstand gefunden, der aus einem Glastrog mit verschiebbaren Bleiblechen bestand. Der Glastrog wurde gefüllt mit Wasser, das durch einige Tropfen verdünnter Schwefelsäure angesäuert worden war. Durch Verschieben der Bleiplatten gegeneinander lälst sich die Stromstärke genau und sicher regulieren.

Die Ausführung der Analysen fand in dem bekannten Cuassen'schen Apparat statt, dessen Kathodenschale etwa $200 \mathrm{ccm}$ falste. Als Anode verwandte ich zuerst eine Platindrahtspirale von $10 \mathrm{qcm}$ Oberfläche, bei späteren Versuchen eine durchbrochene Eimerelektrode mit einer Gesamtoberfläche von etwa $70 \mathrm{qcm}$.

Mittels einer kleinen Wasserturbine konnte die Anode in Rotation versetzt werden.

Beim Erwärmen des Elektrolyten während der Analysen durch einen Mikrobrenner wurde die Platinschale durch ein untergelegtes Asbestpapier geschützt.

Während der Elektrolyse wurde die Schale mit einer kreisrunden Glasscheibe bedeckt, welche einen bis zu ihrem Mittelpunkte reichenden, ca. $1 \mathrm{~cm}$ breiten Einschnitt besafs. Hierdurch wurde es ermöglicht, dals ein passend aufgehängtes Thermometer während der ganzen Dauer der Elektrolyse in dem Elektrolyten eingetaucht gelassen werden konnte.

Das Ende der Elektrolyse wurde durch Prüfen eines herausgenommenen Tropfens der Lösung mit Schwefelwasserstoffwasser erkannt.

Nachdem alles Wismut ausgefällt war, wurde mittels eines Hebers unter fortwährendem Zuflielsenlassen reinen Wassers ohne Stromunterbrechung ausgewaschen, mit Alkohol, dann mit Äther nachgespült und die Schale über einer ganz kleinen Bunsenflamme vorsichtig bis zur völligen Verdunstung des Äthers schwach erwärmt, erkalten gelassen und gewogen.

Um die Schale wieder gebrauchsfertig zu machen, wurde der Metallniederschlag in konzentrierter Salpetersäure gelöst, die Schale ausgespült, getrocknet und über der etwas rauschenden Flamme eines Teclubrenners sorgfältig ausgeglüht. Dieses Ausglühen ist vor 
jedesmaligem Gebrauch der Schale vorzunehmen, weil sonst kein gleichmälsiger Metallüberzug erhalten werden kann.

Die Wismutelektrolyse lälst sich in der gewöhnlichen blanken Classenschale ausführen, jedoch ist eine mattierte Schale vorzuziehen, weil in einer solchen das abgeschiedene Metall besser haftet und sich gleichmäfsiger verteilt als in der blanken Schale.

Die Anode ist genau zu centrieren, weil sich sonst leicht dunkle Stellen im Niederschlag bilden, an denen das Metall sich weniger dicht abscheidet. Derselbe Übelstand tritt auf, wenn die Kathode mit ihrer Unterlage keinen gleichmälsigen Kontakt hat.

\section{Hauptteil.}

Von denjenigen Metallen, deren Nachweis durch Elektrolyse schon früh gelungen ist, ist das Wismut eines der ersten.

Im Jahre 1840 wies CozzI ${ }^{1}$ in tierischen Flüssigkeiten aufser Kupfer, Blei, Antimon und Silber auch Wismut nach mittels einer galvanischen Kette, welche aus einem Gold- und einem Zinkblatte bestand.

1862 beschrieb NickLEs ${ }^{2}$ eine Methode zur Auffindung derselben Metalle und 1865 Luckow $^{3}$ den Nachweis von Silber und Wismut.

Quantitative Bestimmungen finden sich erst später, obgleich schon 1856 die Prinzipien der quantitativen Elektrolyse von MagNus ${ }^{4}$ klar ausgesprochen worden waren.

Im Jahre 1880 brachte die Berg- und Hüttenmännische Zeitung ${ }^{5}$ einen Aufsatz von LuDwia SchuchT, in welchem dieser eine Übersicht über das Verhalten der Metalle bei der Elektrolyse giebt.

Unter denjenigen Metallen, deren quantitative Bestimmung als möglich bezeichnet wird, findet sich zwar das Wismut noch nicht aufgeführt, jedoch enthalten Sсншснт's Mitteilungen zum ersten Male etwas Näheres über das Verhalten des Wismuts bei der Elektrolyse, z. B. dafs es aus saurer und alkalischer Lösung teils als Metall, teils als Superoxyd erhalten werden könne, dafs das Superoxyd im Gegensatz zu dem des Bleis, des Silbers und des Thalliums nur

${ }^{1}$ Arch. delle seienze med. fis. 50, Sem. II, 208.

${ }^{2}$ Kopp u. WrLL, Jahresbericht 1862, 610.

${ }^{3}$ Drnalers Polyt. Journ. 1865, 177, 231; 178, 42.

4 Ber. d. kgl. Akad. Wissensch. Berlin 1856, 158 und Pogg. Ann. 102, 1-54, vergl. überhaupt Ahrens, Handb. d. Elektroch. S. 221-223.

5 Ebendas. 39, 121. 
allmählich sich bilde, dafs in saurer Lösung viel, in alkalischer wenig Superoxyd gebildet werde u. s. w.

Von dem Zeitpunkte dieser Veröffentlichung datiert eine ganze Reihe von Versuchen zur quantitativen Bestimmung des Wismuts durch Elektrolyse, von denen die wichtigsten hier besprochen werden sollen.

Schon in dem der Luckow'schen Veröffentlichung folgenden Jahre sehen wir CLASSEN und ReIss gemeinschaftlich bemüht, die Schwierigkeiten, welche die elektrolytische Bestimmung des Wismuts bietet, zu überwinden; nach mannigfachen Versuchen mit verschiedenen Lösungen berichten sie, ${ }^{1}$ dals es ihnen gelungen sei, die Analyse quantitativ durchzuführen. Jedoch entbehrt die von ihnen angegebene Methode noch sehr der Sicherheit, weil, wie aus ihrem Bericht hervorgeht, sich zuweilen beim Auswaschen Metallteilchen von dem Niederschlag ablösen, welche auf gewogenem Filter gesammelt und besonders bestimmt werden müssen.

$\mathrm{Zu}$ ihren Versuchen wandten ClasSEN und ReISS eine ,mit einem ziemlichen Überschuls von oxalsaurem Ammon versetzte salpetersaure Wismutlösung" an. Über Stromstärke, Stromquellen, Temperatur and Dauer der Analyse machen sie keinerlei Angaben. Ich habe gefunden, dafs Mengen bis $0.2 \mathrm{~g}$ Wismut in 6-8 Stunden bei einer Stromstärke von $0.03-0.05$ Amp. ${ }^{2}$ und einer Spannung von 2 Volt vollständig ausgefällt werden können. Als günstigste Temperatur habe ich $50^{\circ}$ festgestellt.

Erwärmt man auf eine höhere Temperatur, so beginnt sich der Niederschlag stellenweise wieder zu lösen, und bei niederer Temperatur mufs man, um einen einigermalsen festen Niederschlag zu erhalten, die Stromstärke so gering wählen, dals die Analyse bis zu 24 Stunden und länger dauert.

Dals bei Gegenwart von überschüssigem Ammonoxalat zeitweise Superoxyd auftritt, habe ich entgegen den Erfahrungen von CLASSEN und ReIss nicht beobachten können.

Was die Beschaffenheit des Metallniederschlages anbetrifft, so habe auch ich häufig derart schwammige Ausscheidungen erhalten, dafs beim Auswaschen durch Losreifsen von Metallteilchen Verluste eintraten und das erhaltene Wismut nur unter besonderen Kautelen

\footnotetext{
1 Ber. deutsch. chem. Ges. 14 (1881), 1626.

${ }^{2}$ Vergl. Wieland, Ber. deutsch. chem. Ges. 17, 1612.
} 
zur Wägung gebracht werden konnte. Die Methode ist daher als ungeeignet anzusehen.

Zwei Jahre später (1883) traten zwei amerikanische Forscher, N. Whitex Thomas und Edgar F. Smith, ${ }^{1}$ mit verschiedenen Vorschlägen an die Öffentlichkeit, die aber, in unserer deutschen Fachlitteratur wenigstens, wenig Beachtung fanden. Die erste von ihnen vorgeschlagene Methode ist die, Wismut aus einer Lösung in Schwefelsäure abzuscheiden. Über die Stromverhältnisse ist nur angegeben, dal's eine dreizellige, ein Liter fassende Bichromatbatterie benutzt worden sei. Uber die Herstellung der Lösung heifst es in der Vorschrift von Tromas und Smith: „Eine abgewogene Menge von $\mathrm{Bi}_{2} \mathrm{O}_{3}$ wurde in ca. $1 \mathrm{ccm}$ konzentrierter Schwefelsäure gelöst und mit destilliertem Wasser auf $100 \mathrm{ccm}$ verdünnt; $10 \mathrm{ccm}$ dieser Lösung enthielten $0.0358 \mathrm{~g} \mathrm{Wismut".} \mathrm{Trotz} \mathrm{vieler} \mathrm{Versuche} \mathrm{ist} \mathrm{es} \mathrm{mir}$ nicht gelungen, Wismutoxyd mit auch nur annähernd der angegebenen Menge konzentrierter Schwefelsäure in Lösung zu bringen, auch nicht durch mehrtägiges Kochen. Eine spätere Veröffentlichung von Edgar F. Smith und E. B. KNerR ${ }^{2}$ (1886) bringt auch keine näheren Angaben über die Herstellung der Sulfatlösung; es ist dort nur gesagt, dafs die Verfasser die Versuche von Thomas und Sмrтн geprüft und bestätigt gefunden hätten, dals Wismut aus schwefelsaurer Lösung bei Gegenwart freier Schwefelsäure vollständig und schnell ausgefällt werden könne.

Dagegen machen Sмгтн und Knerr Angaben über Dauer, Stromstärke und Temperatur. Sie fällten in einem kleinen Platintiegel mit einem Flüssigkeitsvolumen von $25 \mathrm{ccm}$ bei $0.07 \mathrm{Amp}$. Mengen von ca. $0.02 \mathrm{~g}$ Wismut in $1 \frac{1}{2}$ Stunden bei gewöhnlicher Temperatur vollständig aus. Bei anderen Versuchen mit geringeren Mengen, $0.004-0.008 \mathrm{~g}$ Wismut, wandten sie Stromstärken bis zu 0.4 Amp. an.

Über die Beschaffenheit des ausgeschiedenen Wismuts geben weder Thomas und Suith noch Sмith und KrerR etwas an.

Um das Verhalten des Wismuts bei der Elektrolyse seines Sulfats festzustellen, bereitete ich nach einer von Fresenrus gegebenen Vorschrift ${ }^{3}$ eine Wismutauflösung, indem ich eine abgewogene Menge Wismutoxyd in Salpetersäure löste, unter Zusatz von etwas kon-

I Am. chem. Journ. 5, 114.

2 Am. chem. Journ. 8, 206.

${ }^{3}$ Fresenids, Qualitative Analyse, 15. Auf., S. 182. 
zentrierter Schwefelsäure eindampfte und die zurückbleibende syrupdicke Masse in verdünnter Schwefelsäure auflöste; die so erhaltene Lösung zersetzt sich erst nach längerem Stehen unter Bildung basischer Salze.

Meine mit dieser Wismutsulfatlösung angestellten Versuche hatten wesentlich andere Resultate als die von den obengenannten Autoren angegebenen; insbesondere war das bezüglich der Dauer der Elektrolyse der Fall.

Während z. B. Thomas und Smitr $0.0358 \mathrm{~g}$ Wismut in 3 Stunden ausfällten, dauerte ein von mir unter den gleichen Verhältnissen - soweit dies bei den ungenügenden Angaben dieser Forscher möglich war - angestellter Versuch 24 Stunden. Erst bei höherer Temperatur, bei $45-50^{\circ}$, ging die Fällung schneller vor sich. Es wurden bei $0.02 \mathrm{Amp} .0 .0570 \mathrm{~g} \mathrm{Wismut} \mathrm{in} 5$ Stunden fest und mit dunkelgrauer Farbe ausgeschieden. Bei mehr als 0.05 Amp. erhielt ich stets lockere und schwammige Abscheidungen. Desgleichen fielen grölsere Mengen als etwa $0.05 \mathrm{~g}$ Wismut auch bei geringen Stromstärken gegen Ende der Analyse stets locker und in unbrauchbarer Form aus.

Superoxydbildung wurde in keinem Falle beobachtet.

Aus dem Gesagten ergiebt sich, dafs diese Methode in der Form, wie sie Thomas, Smrth und KnerR vorgeschlagen haben, wegen der Schwierigkeiten, welche ihre Ausführung bietet, kaum brauchbar ist, und ferner, dafs überhaupt die. Verwendung von Schwefelsäure bei der Elektrolyse des Wismuts als unzwekmälsig erscheint, weil dabei nur geringe Mengen des Metalls festhaftend ausgeschieden werden können und die Analyse nach Sмrтн und KNERR anstatt in der gebräuchlichen Classenschale in einem kleinen Platintiegel vorgenommen werden muls.

(S. Tabelle, S. 8.)

Nach einer zweiten Methode fällen Thovas und Smrre Wismut aus einer alkalisch gemachten Citratlösung. Ihren Angaben gemäls wird „Wismuthydroxyd in konzentrierter Citronensäure gelöst und die Lösung mit Natronlauge alkalisch gemacht". „Die Menge des metallischen Wismuts in der Lösung war bekannt und dann wurden bestimmte Mengen der Lösung elektrolysiert." In drei Stunden wurden $0.0358 \mathrm{~g} \mathrm{Bi}$ vollständig ausgefällt, bei einem Strom, welchen "zwei Bichromatzellen" lieferten.

Auch bei dieser Methode besteht die Schwierigkeit, dals eine 
Versuche mit Wismutsulfatlösungen bei Gegenwart von freier Schwefelsäure.

\begin{tabular}{c|c|c|c|c|c|c|c|c}
\hline $\begin{array}{c}\text { An- } \\
\text { gewandt }\end{array}$ & $\begin{array}{c}\text { Ge- } \\
\text { funden } \\
\mathrm{g}\end{array}$ & $\begin{array}{c}\mathrm{V} \text { - } \\
\mathrm{lum} \\
\mathrm{ccm}\end{array}$ & $\begin{array}{c}\mathrm{H}_{2} \mathrm{SO}_{4} \\
\mathrm{ccm}\end{array}$ & $\begin{array}{c}\text { Temp. } \\
0\end{array}$ & $\begin{array}{c}\text { Strom- } \\
\text { stärke } \\
\text { Amp. }\end{array}$ & $\begin{array}{c}\text { Dauer } \\
\text { Stunden }\end{array}$ & $\begin{array}{c}\text { Nieder- } \\
\text { seblag } \\
\text { Qualität }\end{array}$ & $\begin{array}{c}\text { Diffe- } \\
\text { renz } \\
\%\end{array}$ \\
\hline 0.0358 & 0.0358 & $25 ?$ & 1 & $20 ?$ & - & 3 & - & $0.03^{*}$ \\
0.0229 & 0.0229 & 25 & 1 & $20 ?$ & 0.08 & $11 / 2$ & - & $0.03^{*}$ \\
0.0282 & - & 25 & 1 & 20 & 0.02 & 24 & fest & - \\
0.0330 & 0.0326 & 150 & 1 & 20 & 0.02 & 28 & fest & -1.3 \\
0.0592 & 0.0570 & 25 & 1 & 50 & 0.02 & $4^{3 / 4}$ & fest & -3.5 \\
0.0516 & - & 150 & 1 & 50 & 0.03 & - & schwamm. & - \\
0.0577 & - & 150 & ca. 10 & 50 & 0.03 & - & schwamm. & - \\
& & & & & & &
\end{tabular}

* Die erste Analyse ist von Tromas und Sмrтr, die zweite von Sмmrr und KNERR ausgeführt und zum Vergleich beigefügt.

Lösung einer vorher abgewogenen Menge der Wismutverbindung nicht herzustellen ist, weil getrocknetes Wismuthydroxyd in Citronensäure schwer löslich ist. Man mnfs deshalb mit empirischen Lösungen arbeiten oder den Wismutgehalt zur Kontrolle auf anderem Wege feststellen. Dieser Sinn scheint auch in den Worten der oben angeführten Überschrift: „die Menge des metallischen Wismuts in der Lösung war bekannt", zu liegen.

Ein fernerer Übelstand bei dieser Methode ist das starke Auftreten von Superoxyd an der Anode, welches im Laufe der Analyse nicht wieder verschwindet, mithin die Bestimmung des Wismuts unmöglich macht.

NB. Nach meinen Erfahrungen tritt überhaupt in alkalischen und auch ammoniakalischen Lösungen das Wismutsuperoxyd in stärkerem Malse auf als in sauren Lösungen, während ScHणCHт, ${ }^{x}$ gerade umgekehrt angiebt, dafs in sauren Lösungen viel, in alkalischen wenig Superoxyd sich bilde.

Ferner war bei Versuchen, die ich mit empirischen Lösungen unter Variierung von Temperatur und Alkaligehalt anstellte, der Metallniederschlag stets schwarz und locker, also unbrauchbar. Nur bei ganz geringen Mengen von Wismut, wie sie Thомas und Sмrтн anwandten, $0.03-0.04 \mathrm{~g}$, liefsen sich einigermalsen gute Nieder-

1 Berg- u. Hüttenmänn. Zeitschr. 39, 121 und Zeitschr. analyt. Chem. 22 (1883). 
schläge erhalten. Im ganzen mufs jedoch auch diese Methode in der angegebenen Form als zur allgemeinen Anwendung ungeeignet bezeichnet werden.

Versuche mit alkalisch gemachten Wismutcitratlösungen.

\begin{tabular}{c|c|c|c|c|c|c|c}
\hline $\begin{array}{c}\text { An- } \\
\text { gewandt } \\
\mathrm{g}\end{array}$ & $\begin{array}{c}\text { Ge- } \\
\text { funden } \\
\mathrm{g}\end{array}$ & $\begin{array}{c}\text { Temp. } \\
0\end{array}$ & $\begin{array}{c}\text { Strom- } \\
\text { stärke } \\
\text { Amp. }\end{array}$ & $\begin{array}{c}\text { Dauer } \\
\text { Stunden }\end{array}$ & $\begin{array}{c}\text { Nieder- } \\
\text { schlag } \\
\text { Qualität }\end{array}$ & $\begin{array}{c}\text { Super- } \\
\text { oxyd }\end{array}$ & $\begin{array}{c}\text { Diff. } \\
\%\end{array}$ \\
\hline 0.0356 & 0.0358 & 20 & - & 3 & fest & keins & $+0.6^{*}$ \\
0.0549 & - & 20 & 0.05 & - & schwammig & $\begin{array}{c}\text { viel } \\
\text { keins }\end{array}$ & \\
0.0382 & - & 20 & 0.05 & 8 & $n$ & $n$ \\
0.0382 & - & 45 & 0.05 & - & $n$ & viel & \\
0.0549 & - & 20 & 0.03 & - & $n$ & sehr viel &
\end{tabular}

* Die erste der angeführten Analysen ist den Beleganalysen von Thomas und SMrre entnommen.

Der dritte Vorschlag endlich, den Thomas und Sмrrн machen, ist der, eine weinsaure, mit Natronlauge alkalisch gemachte und wieder mit Citronensäure angesäuerte Wismutlösung zu verwenden. Aus dieser Lösung sollen in der Kälte $0.0357 \mathrm{~g} \mathrm{Wismut} \mathrm{in} 2^{1} / 2$ Stunden ausfallen.

Als Stromquellen werden wieder ,zwei Bichromatzellen" angegeben, während über die Stromstärke Näheres nicht gesagt ist. Erwärmen soll die Fällung nicht beschleunigen und Superoxyd zwar zeitweise auftreten, aber während der Elektrolyse wieder verschwinden.

Nach der von Thomas und Smi'r angegebenen Vorschrift löste ich Wismutoxyd in Weinsäure, machte mit Natronlange alkalisch und säuerte mit Citronensäure an. Hierbei erhielt ich meistens eine klare Lösung, welche ich auf $150 \mathrm{ccm}$ verdünnte und einem Strome von $0.01-0.05 \mathrm{Amp}$. aussetzte. Die Spannung betrug wie bei den früheren Versuchen zwei Volt.

Die Versuche fielen insofern günstiger aus als bei den beiden früheren Versuchen, als kein Superoxyd an der Anode auftrat. Der Metallniederschlag war jedoch bei Zimmertemperatur selbst bei Anwendung ganz geringer Mengen stets schwarz und schwammig. Erwärmen auf ca. $50^{\circ}$ hatte bei meinen Versuchen auf die Beschaffenheit des Niederschlages einen günstigen Einflufs, so dals bei Strömen von $0.03 \mathrm{Amp}$. brauchbare Ausscheidungen erzielt werden konnten. 
Wismutoxyd in Weinsäure zu lösen, gelingt, wie oben angedeutet, nicht leicht. Hierin sowie in der Notwendigkeit, die geringe Stromstärke mit peinlicher Genauigkeit einzuhalten, liegt die hauptsächliche Schwäche der Methode, welche mithin zur Ausführung nicht empfohlen werden kann, was auch in den mir zugänglichen Handbüchern für Elektroanalyse nirgends geschehen ist.

Um die Schwierigkeiten der Lösung ron Wismutoxyd in Weinsäure zu umgehen, benutzte ich bei meinen nachfolgend aufgeführten Versuchen festes Wismuttartrat, das in kalter Natronlauge leicht löslich ist, jedoch nicht den berechneten Wismutgehalt besitzt. Daher wurden viel zu hohe Werte für das gefundene Wismut erhalten.

Versuche mit alkalisch gemachten und mit Citronensäure wieder angesäuerten Wismuttartratlösungen.

\begin{tabular}{c|c|c|c|c|c|c|c}
\hline $\begin{array}{c}\text { An- } \\
\text { gewandt }\end{array}$ & $\begin{array}{c}\text { Ge- } \\
\text { funden } \\
g\end{array}$ & $\begin{array}{c}\text { Strom- } \\
\text { stärke } \\
\text { Amp. }\end{array}$ & $\begin{array}{c}\text { Dauer } \\
\text { Stunden }\end{array}$ & $\begin{array}{c}\text { Temp. } \\
0\end{array}$ & $\begin{array}{c}\text { Nieder- } \\
\text { schlag } \\
\text { Besch. }\end{array}$ & $\begin{array}{c}\text { Super- } \\
\text { oxyd }\end{array}$ & $\begin{array}{c}\text { Diff. } \\
\%\end{array}$ \\
\hline-0.0356 & 0.03564 & - & $21 / 2$ & 20 & $\#$ & $\begin{array}{c}\text { ver- } \\
\text { schwind. }\end{array}$ & $+0.1^{*}$ \\
0.0359 & - & 0.03 & - & 20 & schwammig & keins & - \\
0.0374 & 0.0359 & 0.02 & $31 / 2$ & 55 & fest & $"$ & -5.5 \\
0.0507 & 0.0547 & 0.03 & $21 / 2$ & 55 & locker & $"$ & +8.0 \\
0.1260 & 0.1354 & 0.03 & 5 & 55 & fest & $"$ & +7.0 \\
0.1602 & - & 0.06 & $61 / 2$ & 20 & locker & $"$ & -
\end{tabular}

* Die in der ersten Reihe wiedergegebenen Zahlen stellen die Mittelwerte der Beleganalysen von Thomas und Sмrтt dar.

In demselben Jahre, in welchem die drei zuletzt besprochenen Methoden veröffentlicht worden waren, trat CLassen in Gemeinschaft mit Ludwig und Eliasberg ${ }^{1}$ mit einem neuen Vorschlag hervor. Auf den früheren Versuchen von Classen und ReIss (vergl. S. 1) fufsend, wandten sie wiederum die Oxalsäure an in Form ihres Wismutammondoppelsalzes. Nach ihrer von ELLIASBERG im einzelnen angegebenen Vorschrift wird das in tarierter Platinschale abgewogene Wismutsalz mit ca. $10 \mathrm{ccm}$ Kaliumoxalatlösung, welche etwa $30 \%$ des Salzes enthält, versetzt und nach und nach festes Ammonoxalat zugefügt, bis völlige Lösung eintritt. Dann verdünnt man auf etwa $150 \mathrm{~cm}$, erhitzt auf $70-80^{\circ}$, und lärst unter Beibehaltung dieser

1 Ber. deutsch. chem. Oes. 19 (1886), 1326. 
Temperatur den elektrischen Strom einwirken. Nach sechzehn Stunden wird reine Oxalsäure bis zur sauren Reaktion zugegeben und die Elektrolyse fortgesetzt. Ist nach vierundzwanzig Standen kein Wismut mehr in der. Lösung nachzuweisen, so wäscht man ohne Stromunterbrechung aus, trocknet und wägt.

Dem Aussehen nach soll das auf diese Weise abgeschiedene Metall schön hell und krystallinisch sein und sehr fest an der Schale haften. Jedoch fallen die Resultate infolge partieller Oxydation oft zu hoch aus. Bei Mengen von durchschnittlich $0.17 \mathrm{~g}$ Wismut zeigen die Beleganalysen Differenzen bis zu $+0.7 \%$. Dieser Ungenauigkeit will EuIASBERG dadurch begegnen, dafs er den Niederschlag in der Schale mit Salpetersäure löst, auf dem Wasserbade eindampft und durch Glühen in Wismutoxyd überführt, das alsdann zur Wägung gebracht wird. Dieses Verfahren ist jedoch milslich, einerseits, weil dadurch die Zahl der Manipulationen, mithin die der Fehlerquellen vermehrt wird, anderseits deshalb, weil das Wismutnitrat beim Glühen die Neigung zeigt, zu verspritzen, so dafs diese Operation im ganzen kaum als Vorteil angesehen werden kann.

Ein weiterer Übelstand bei dieser Methode ist die lange Dauer der Analyse, verbunden mit einer verhältnismälsig hohen Temperatur. Die letztere erfordert, auch wenn die Schale mit einem Uhrglas bedeckt ist, einen öfteren Ersatz des verdampfenden Wassers und ferner eine sorgfältige Regulierung des Gasdruckes, da dieser erfahrungsgemäls innerhalb vierundzwanzig Stunden bedeutenden Schwankungen unterworfen ist. Es ist also eine beständige Überwachung nötig, was, da die Analyse zum Teil über Nacht gehen mufs, mit Schwierigkeiten verknüpft und in den meisten Übungslaboratorien nicht ausführbar ist.

Über die Stromverhältnisse ist nur angegeben, dafs der Strom sehr schwach sein müsse und von den Verfassern zwei MeIDINGerElemente mit eingeschalteten sechzig Ohm benutzt worden seien. Die Stromstärke dürfte $0.01 \mathrm{Amp}$. nicht überschritten haben.

Um die unbequem lange Dauer der Elektrolyse abzukürzen, wählte ich bei meinen Versuchen Stromstärken von 0.02 bis $0.03 \mathrm{Amp}$. Im übrigen verfuhr ich nach der vorhin angegebenen Vorschrift; jedoch verwandte ich statt eines Wismutsalzes das Oxyd $\mathrm{Bi}_{2} \mathrm{O}_{3}$. Zur Lösung von $0.1-0.2 \mathrm{~g}$ Wismutoxyd waren 5-10 g Ammoniumoxalat erforderlich.

Aus meinen Versuchen ergab sich, dals Mengen von ca. $0.12 \mathrm{~g}$ Wismut in 8-9 Stunden ziemlich festhaftend ausgeschieden werden. 
Jedoch erhielt auch ich stets zu hohe Werte, und zwar waren die Differenzen oft beträchtlich und stiegen manchmal bis zu mehreren Prozenten. Die Temperatur von $70-80^{\circ}$ mufs eingehalten werden, wenn der Niederschlag die erforderliche Dichte haben soll; ferner darf die Stromstärke von 0.03 Amp. nicht überschritten werden. Je geringer die Stromdichte ist, desto fester und heller wird der Niederschlag und desto langsamer geht die Ausscheidung vor sich. Auftreten von Superoxyd an der Anode habe ich niemals beobachtet.

Als Resultat meiner Untersuchungen über diese Methode ergiebt sich, dafs sie neben minder gewichtigen Unzuträglichkeiten die allzugrofser Ungenauigkeit der erhaltenen Werte aufweist, mithin den Anforderungen, welche an eine gute elektrolytische Methode zu stellen sind, nicht entspricht.

Versuche mit Lösungen von Wismutammonoxalat. ${ }^{1}$

\begin{tabular}{|c|c|c|c|c|c|c|c|}
\hline $\begin{array}{c}\text { Au- } \\
\text { gewandt } \\
g\end{array}$ & $\begin{array}{l}\text { Ge- } \\
\text { funden } \\
\qquad\end{array}$ & Temp. & $\begin{array}{l}\text { Strom- } \\
\text { stärke } \\
\text { Amp. }\end{array}$ & $\begin{array}{c}\text { Dauer } \\
\text { Stunden }\end{array}$ & $\begin{array}{l}\text { Nieder- } \\
\text { schlag } \\
\text { Besch. }\end{array}$ & $\begin{array}{c}\text { Super- } \\
\text { oxyd }\end{array}$ & $\begin{array}{c}\text { Diff. } \\
\%\end{array}$ \\
\hline 0.1567 & 0.1575 & $70-80$ & $\begin{array}{c}\text { sehr } \\
\text { schwach }\end{array}$ & 24 & fest & keins & $+0.5^{*}$ \\
\hline 0.0646 & 0.0686 & 75 & 0.02 & $6^{3} / 4$ & 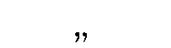 & $"$ & +6.0 \\
\hline 0.0712 & 0.0716 & 75 & 0.02 & 8 & locker & $"$ & +0.57 \\
\hline 0.0753 & 0.0766 & 45 & 0.02 & $63 / 4$ & $\eta$ & 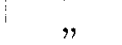 & +1.9 \\
\hline 0.1051 & 0.1086 & 75 & 0.02 & $51 / 4$ & schwammig &, & +3.7 \\
\hline 0.0376 & 0.0986 & 75 & 0.02 & 5 & , & 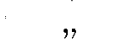 & +1.0 \\
\hline 0.1164 & 0.1172 & 55 & 0.02 & $8^{8} / 4$ & locker & $"$ & +0.7 \\
\hline 0.1221 & 0.1227 & 75 & 0.02 & $8^{3} / 4$ & fest & 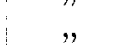 & +0.5 \\
\hline 0.1480 & 一 & $5 \overline{5}$ & 0.02 & 10 & schwammig &, & - \\
\hline 0.1300 & - & 55 & 0.02 & 7 & , & , & - \\
\hline 0.2344 & - & 75 & 0.02 & 8 & " & $"$ & - \\
\hline
\end{tabular}

* Die in dieser Reihe angegebenen Zahlen stellen die von Cuassan, Lonwig und Eliasbera in ibren Beleganalysen mitgeteilten Werte im Mittel dar.

Die bisher besprochenen Methoden kranken alle an dem Fehler, daf's das Lösungsmittel im starken Überschuls angewandt werden mufs, weil die Wismutverbindungen in diesem nicht leicht löslich sind. Nach meinen Beobachtungen ist es aber gerade bei der Elektro-

1 Die bei allen Analysen angegebene Stromstärke von $0.02 \mathrm{Amp}$. ist ein Mittelwert. In Wahrheit schwankte sie zwischen 0.01 und 0.03 Amp. Eine genaue Ablesung auf hundertstel Amp. gestattete das mir damals zur Verfügung stehende Ampèremeter nicht. 
lyse des Wismuts von Vorteil, wenn das Lösungsmittel in möglichst geringer Menge verwendet werden kann, weil sonst das Metall dazu neigt, sich locker abzuscheiden, sobald Stromstärke und angewandte Menge des Wismuts über ein gewisses, sehr geringes Mals hinausgehen.

Ein Lösungsmittel, das in verhältnifsmälsig geringer Menge die Wismutverbindungen leicht und vollständig löst, ist die Salpetersäure. Es ist daher nicht zu verwundern, dafs schon früh Versuche mit salpetersauren Wismutlösungen gemacht worden sind. Alle diese Versuche führten aber zu keinem befriedigenden Resultat, weil Neigung zu Schwammbildung, Auftreten von Superoxyd, lange Dauer, kurz, die ganze Reihe der für die Wismutelektrolyse typischen Schwierigkeiten sich in den Weg stellten.

Trotzdem habe ich mein Hauptaugenmerk auf die Verwendung salpetersaurer Lösungen gerichtet, weil die Schwierigkeiten, die sich dabei bieten, mir eher überwindlich schienen, als die der anderen Methoden.

Wichtige Angaben bezüglich des Verhaltens von Wismut in salpetersaurer Lösung bei der Elektrolyse machte schon 1884 WIELAND, ${ }^{1}$ welcher feststellte, daís es nur bei ganz geringen Strömen, 0.01-0.05 Amp., gelinge, Wismut aus oxalsaurer oder salpetersaurer Lösung in kompaktem Zustande zu erhalten, dals aber dann die Fällung sehr langsam vor sich gehe; ferner fanden 1893 Smrrt und Saltar, ${ }^{2}$ deren Versuche auf die Feststellung der günstigsten Menge Salpetersäure gerichtet waren, dals nur soviel Säure zuzusetzen sei, als zur Verhütung der Bildung basischer Salze erforderlich sei. In diesem Falle erhalte man einen festen Metallniederschlag und vollständige Ausfällung; auch werde kein Superoxyd an der Anode gebildet. Dieses trete erst bei Anwesenheit von mehr freier Salpetersäure auf, wobei auch die Fällung unvollständig sei. Sie arbeiteten mit einer Stromstärke von etwa $0.2 \mathrm{Amp}$.

Diese Angabe steht nicht im Einklang mit den oben erwähnten Erfahrungen WIELAND's. Auch ich habe die Stromstärke von $0.2 \mathrm{Amp}$. zu hoch gefunden; meine Versuche in dieser Richtung milslangen alle, weil das Wismut bei mehr als $0.03 \mathrm{Amp}$. schwammig ausfiel.

Bessere Resultate erhielt ich nach dem folgenden Verfahren:

1 Ber. deutsch. ohem. Ges. 17, 1612.

2Z. anorg. Chem. 3, 416. 
Ich löste Wismutoxyd in abgewogener Menge in konzentrierter Salpetersäure, und zwar rechnete ich auf je $0.1 \mathrm{~g} \mathrm{Bi}_{2} \mathrm{O}_{3} 0.5-1 \mathrm{ccm}$ Säure. Dann verdünnte ich in einem Melskolben, in welchem auch die Auflösung vorgenommen worden war, auf $250 \mathrm{ccm}$, bei anderen Versuchen auf 500 und $1000 \mathrm{ccm}$. Mit einer Pipette abgemessene Mengen dieser Lösung gab ich in die Classenschale, füllte zu $200 \mathrm{ccm}$ auf und liefs einen Strom von 0.01-0.03 Amp. einwirken. Die Temperatur betrug $40-60^{\circ}$, die Dauer je nach der angewandten Menge (0.05-0.2 g Bi) 6-9 Stunden.

In den meisten Fällen waren die Niederschläge, insbesondere gegen Ende der Analyse, dunkel und locker, wodurch zuweilen beim Auswaschen Verluste eintraten. Auch beobachtete ich, entgegen den Erfahrungen von SMith und SaLtar, jedesmal das Auftreten von Superoxyd, allerdings in nur geringer Menge. Es wurde durch Zusatz von einigen Tropfen Glycerinlösung entfernt bezw. unterdrückt. In Übereinstimmung mit Sнмттн und SALTAR fand ich, dafs Vermehrung des Säuregehaltes die Superoxydbildung befördert. Ferner wächst mit der Säuremenge die Neigung des Metalls, schwammig auszufallen.

Erwärmen beschleunigt die Fällung, jedoch ist eine Temperatur von mehr als $60^{\circ}$ nicht zweckmäfsig, weil dabei der Niederschlag teilweise wieder in Lösung gehen kann.

Unter dem Mikroskop stellt sich der Metallniederschlag als eine aus nadelförmigen Kryställchen bestehende dichte Masse dar. Die Abscheidung erfolgt in der Weise, dafs die Hauptmenge zu Anfang der Elektrolyse niedergeschlagen wird und die Geschwindigkeit der Ausscheidung stetig abnimmt, so dals es zweckmäfsig ist, gegen Ende der Elektrolyse die Stromstärke etwas zu erhöhen, um die letzten Anteile schneller zur Ausscheidung zu bringen. Bei einer Gesamtdauer von 8 Stunden wurde bei einem Versuch mit $0.2 \mathrm{~g}$ Wismut in der ersten Stunde $0.1 \mathrm{~g}$, in der zweiten $0.04 \mathrm{~g}$, in der dritten $0.02 \mathrm{~g}$ Wismut ausgeschieden. Trägt man die Dauer als Abscisse, die ausgeschiedenen Mengen in Gramm als Ordinate in ein Koordinatensystem ein, so erhält man eine anfangs stark ansteigende, dann rasch sich verflachende Kurve.

In demselben Malse, als die Geschwindigkeit der Ausscheidung abnimmt, nimmt auch die Dichte und Festigkeit des Metallniederschlages ab, so dafs dieser gegen Ende oft schwammig und locker ausfällt.

Versuche, diesem Übelstand durch Neutralisation der während 
der Elektrolyse frei gewordenen Säure mit Natron abzuhelfen, blieben ohne Erfolg. Dagegen wurde durch den Zusatz des Alkalis die Menge des entstehenden Superoxyds vermehrt.

In einer anderen Reihe von Versuchen, bei welchen ein Teil der Salpetersäure durch organische Säuren ersetzt war, erhielt ich bessere, aber doch keine befriedigenden Resultate. Auch Zusatz von Glycerin in grölseren Mengen brachte keinen erheblichen Vorteil.

Versuche mit salpetersauren Wismutlösungen ohne Zusatz.

\begin{tabular}{|c|c|c|c|c|c|c|c|}
\hline $\begin{array}{c}\text { An- } \\
\text { gewandt } \\
g\end{array}$ & $\begin{array}{l}\text { Ge- } \\
\text { funden } \\
\quad \mathrm{g}\end{array}$ & $\begin{array}{c}\mathrm{HNO}_{3} \\
\mathrm{ecm} .\end{array}$ & $\begin{array}{c}\text { Temp. } \\
0\end{array}$ & $\begin{array}{l}\text { Strom- } \\
\text { stärke } \\
\text { Amp. }\end{array}$ & $\begin{array}{c}\text { Dauer } \\
\text { Stunden }\end{array}$ & $\begin{array}{l}\text { Nieder- } \\
\text { schlag } \\
\text { Besch. }\end{array}$ & $\begin{array}{c}\text { Diff. } \\
\%\end{array}$ \\
\hline 0.0826 & 0.0820 & $\ldots$ & 20 & $0.01-0.03$ & $25 \%$ & fest & -0.75 \\
\hline 0.1190 & 0.1194 & - & 20 & $0.01-0.03$ & 17 & , & +0.35 \\
\hline 0.1252 & 0.1252 & - & 20 & $0.01-0.03$ & 22 & 3 & 0.00 \\
\hline 0.1312 & 0.1308 & - & 20 & $0.01-0.03$ & 21 & $"$ & -0.31 \\
\hline 0.0593 & 0.0593 & - & - & ca. 0.2 & - & $"$ & $0.00^{*}$ \\
\hline 0.0496 & 0.0489 & $1 / 2$ & 45 & $0.01-0.03$ & $8^{1} / 2$ & 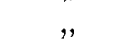 & -1.36 \\
\hline 0.0500 & 0.0492 & $1 / 2$ & 45 & $0.01-0.03$ & $8^{1 / 4}$ & 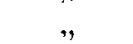 & -2.0 \\
\hline 0.1001 & 0.1007 & 1 & 45 & $0.01--0.03$ & $71 / 2$ & dunkel & +0.7 \\
\hline 0.1001 & 0.0999 & 1 & 45 & $0.01-0.03$ & $81 / 2$ & fest & -0.2 \\
\hline 0.1972 & 0.1972 & 2 & 75 & $0.01--0.03$ & $71 / 2$ & hell, fest & 0.00 \\
\hline 0.3944 & 0.3884 & 4 & 75 & $0.01-0.03$ & 6 & fest & -2.0 \\
\hline 0.3944 & 0.3937 & 4 & 75 & $0.01-0.03$ & $5^{3 / 4}$ & locker & -0.12 \\
\hline 0.3944 & 0.3951 & 4 & 75 & $0.01-0.03$ & $81 / 2$ & fest & +0.18 \\
\hline 0.1972 & 0.1973 & 2 & 75 & $0.01-0.01$ & 5 & dunkel & +0.05 \\
\hline
\end{tabular}

* Smith und Salmar.

Versuche mit salpetersaurer Wismutlösung unter Zusatz von Eisessig.

\begin{tabular}{|c|c|c|c|c|c|c|c|c|}
\hline $\begin{array}{c}\text { An- } \\
\text { gewandt } \\
g\end{array}$ & $\begin{array}{l}\text { Ge- } \\
\text { funden } \\
\quad \mathrm{g}\end{array}$ & $\begin{array}{c}\mathrm{HNO}_{\mathrm{g}} \\
\mathrm{ccm}\end{array}$ & $\begin{array}{l}\text { Eis- } \\
\text { essig } \\
\text { ecm }\end{array}$ & $\begin{array}{c}\text { Temp. } \\
0\end{array}$ & $\begin{array}{c}\text { Strom- } \\
\text { stärke } \\
\text { Amp. }\end{array}$ & $\begin{array}{c}\text { Dauer } \\
\text { Stunden }\end{array}$ & $\begin{array}{l}\text { Nieder- } \\
\text { schlag } \\
\text { Besch. }\end{array}$ & $\begin{array}{c}\text { Diff. } \\
\%\end{array}$ \\
\hline 0.0372 & - & 0.4 & 0.5 & 20 & 0.01 & $16^{1 / 2}$ & dunkel & - \\
\hline 0.0372 & - & 0.4 & 1 & 55 & 001 & & & - \\
\hline 0.0496 & 0.0489 & 0.5 & 0.2 & 45 & $0.01-0.03$ & $6^{1 / 4}$ & fest & -1.4 \\
\hline 0.1000 & 0.1002 & 0.25 & 0.2 & 50 & $0.01-0.03$ & $6 \frac{1}{2}$ & " & +1. \\
\hline 0.1000 & 0.1000 & 0.25 & 0.25 & 50 & $0.01-0.03$ & $71 / 2$ & ", & 0. \\
\hline 0.2001 & 0.2000 & 0.5 & 0.15 & 50 & $0.01-0.03$ & 9 & & -0 \\
\hline 0.2015 & 0.2014 & 0.5 & 0.5 & 55 & $0.01-0.03$ & $6 \%$ & - & -0.0 \\
\hline 0.2015 & 0.1969 & 0.5 & 0.3 & 55 & $0.01-0.03$ & $51 / 4$ & dunkel & -2.3 \\
\hline
\end{tabular}

NB. Es bildet sich jedesmal ein dünner von Superoxyd auf der Anode. 
Versuche mit salpetersaurer Wismutlösung unter Zusatz von Ameisensäure.

\begin{tabular}{|c|c|c|c|c|c|c|c|c|}
\hline $\begin{array}{c}\text { An- } \\
\text { gewandt } \\
g\end{array}$ & $\begin{array}{l}\text { Ge- } \\
\text { funden } \\
\qquad g\end{array}$ & $\begin{array}{c}\mathrm{HNO}_{3} \\
\mathrm{ecm}\end{array}$ & $\begin{array}{c}\mathrm{HCOOH} \\
\mathrm{ecm} \\
(25 \%)\end{array}$ & Temp. & $\begin{array}{l}\text { Strom- } \\
\text { stärke } \\
\text { Amp. }\end{array}$ & $\begin{array}{c}\text { Dauer } \\
\text { Stda. }\end{array}$ & $\begin{array}{l}\text { Nieder- } \\
\text { schlag } \\
\text { Besch. }\end{array}$ & $\begin{array}{c}\text { Diff. } \\
\%\end{array}$ \\
\hline 0.0558 & 0.0555 & 0.3 & 1.5 & 45 & 0.01 & 6 & hellgrau & -0.55 \\
\hline 0.0558 & 0.0564 & 0.3 & 12 & $4 \tilde{5}$ & $0.01-0.03$ & 7 & dunkel & +1.10 \\
\hline 0.0558 & 0.0558 & 0.3 & 14.5 & 45 & $0.01-0.03$ & 7 & hellgrau & 0.4 \\
\hline 0.0558 & 0.0564 & 0.3 & 14.5 & 45 & $0.01-0.03$ & 7 & , & +1.10 \\
\hline 0.0558 & 0.0554 & 0.3 & 16 & 45 & $0.01-0.03$ & 7 & $\Rightarrow$ & -0.74 \\
\hline 0.0623 & - & 0.3 & 7.2 & 20 & 0.01 & 23 & dunkel & - \\
\hline 0.1000 & 0.1008 & 0.5 & 1 & 45 & $0.01-0.03$ & $61 / 2$ & dunkel & +0.8 \\
\hline
\end{tabular}

NB. Bei Gegenwart von Ameisensäure wurde kein Superoxyd gebildet.

Der Niederschlag war jedesmal fleckig.

Versuche mit salpetersauren Wismutlösungen unter Zusatz von gröfseren Mengen Glycerin.

\begin{tabular}{c|c|c|c|c|c|c|c}
\hline $\begin{array}{c}\text { An- } \\
\text { wandte }\end{array}$ & $\begin{array}{c}\text { Ge- } \\
\text { funden }\end{array}$ & $\begin{array}{c}\text { Glycerin } \\
\mathrm{g}\end{array}$ & $\begin{array}{c}\text { Temp. } \\
\mathrm{ecm}\end{array}$ & $\begin{array}{c}\text { Strom- } \\
\text { stärke } \\
\text { Amp. }\end{array}$ & $\begin{array}{c}\text { Nieder- } \\
\text { schlag } \\
\text { Besch. }\end{array}$ & $\begin{array}{l}\text { Dauer } \\
\text { Stdn. }\end{array}$ & $\begin{array}{c}\text { Diff. } \\
\%\end{array}$ \\
\hline 0.0992 & 0.0923 & 30 & 50 & $0.01-0.03$ & hell, fest & $6 / 4$ & -7.7 \\
0.0992 & 0.1037 & 25 & 50 & $0.01-0.03$ & unrein, fest & $1 ;$ & +5.0 \\
0.0992 & 0.1001 & 30 & 50 & $0.01-0.03$ & hell, fest & 7 & +0.9 \\
0.1094 & 0.1087 & 30 & 50 & $0.01-0.03$ & hell, fest & $6 \mathbf{1} / 2$ & -0.6 \\
0.0992 & 0.0993 & 30 & 50 & $0.01-0.03$ & hell, fest & $61 / 2$ & +0.1 \\
0.1959 & 0.1955 & 5 & 50 & $0.03-0.04$ & hell, fest & 6 & -0.2 \\
0.1985 & - & 30 & 55 & $0.01-0.03$ & schwammig & 6 & -
\end{tabular}

NB. Superoxyd wurde nicht gebildet.

Die Glycerinlösung bestand aus 1 Teil käuflichem Glycerin und 2 Teilen Wasser.

Bei all diesen Versuchen fielen die Resultate ungleichmälsig und unsicher aus. Ich gab es daher auf, durch irgend welche Zusätze zu der Wismutlösung zum Ziele zu gelangen, sondern wählte einen ganz anderen Weg.

Ich stellte Wismutlösungen her, die auf $0.1 \mathrm{~g} \mathrm{Bi}_{2} \mathrm{O}_{3} 1-0.5 \mathrm{ccm}$ konzentrierte Salpetersäure enthielten und elektrolysierte abgemessene Mengen davon mit Strömen von wenigstens 0.05 Amp. unter Bewegung des Elektrolyten, welche durch die auf Seite 3 beschriebene Vorrichtung bewirkt wurde. In dieser Weise ausgeführt, erforderte 
die Analyse kaum die Hälfte der Zeit, welche bei den Versuchen ohne Bewegung des Elektrolyten nötig war, und der Niederschlag war bei Einhaltung der unten näher zu besprechenden Bedingungen stets fest und liefs sich ohne Verlust auswaschen. Ein weiterer Vorzug des Arbeitens mit bewegtem Elektrolyten ist der, dafs sich in salpetersaurer Lösung kein Superoxyd an der Anode bilden kann, weil diese durch die Bewegung immer wieder mit frischen Säureteilchen in Berührung gebracht wird und so das Superoxyd bei seiner leichten Löslichkeit auch in verdünnter Salpetersäure im Entstehen stets wieder gelöst wird, während sich bei ruhender Anode um diese herum eine schützende Gasschicht bilden kann, welche das Ansetzen von Superoxyd begünstigt.

Auf diese Weise gelingt es, Mengen bis etwa $0.4 \mathrm{~g}$ Wismut mit hinreichender Genauigkeit und Sicherheit auszuscheiden. Die Dauer beträgt 3-4 Stunden bei einer Stromstärke von 0.05 Amp. Um die Dauer noch mehr abzukürzen, kann man zu Anfang der Elektrolyse mit einer höheren Stromstärke arbeiten, als zur Durchführung der ganzen Analyse statthaft wäre, da sich das Wismut zuerst stets sehr dicht und fest ausscheidet und erst, wenn der gröfste Teil des Metalls ausgefällt ist, Neigung zu Schwammbildung zeigt. Man beginnt zweckmärsig mit einer Stromstärke von $0.1 \mathrm{Amp}$. und geht, sobald der Niederschlag sich dunkler zu färben beginnt, was bei mittleren Mengen nach etwa einer Stunde eintritt, auf $0.05 \mathrm{Amp}$. herunter. Diese Stromstärke behält man dann bis zum Ende der Elektrolyse bei.

Bei Anwendung einer innen gerauhten Kathodenschale erhält man hellere und gleichmäfsigere Ausscheidung ais in glatten Schalen (s. S. 4).

Die Temperatur lälst man am besten allmählich bis auf $50^{\circ}$ steigen und behält diese dann bis zur Beendigung der Elektrolyse bei.

Die weitere Behandlung des erhaltenen Wismutniederschlages geschieht wie oben (S. 3) beschrieben.

(S. Tabelle, S. 18.)

Die Spannung betrug stets zwei Volt.

Will man Wismutnitrat als Ausgangsmaterial benutzen, so lälst sich, wie ich ermitteite, als Lösungsmittel das Glycerin mit Vorteil anwenden. Das feste Wismutnitrat löst sich nämlich in einer wässerigen Glycerinlösung leicht und vollständig auf, und zwar in der 2. anorg. Chem. XXVII. 
Versuche mit salpetersauren Wismutlösungen ohne Zusatz bei bewegtem Elektrolyten.

\begin{tabular}{|c|c|c|c|c|c|c|c|}
\hline $\begin{array}{c}\text { An- } \\
\text { gewandt } \\
\text { g } 13 \mathrm{i}\end{array}$ & $\begin{array}{l}\text { Ge- } \\
\text { funden } \\
g \mathrm{Bi}\end{array}$ & $\begin{array}{c}\mathrm{HNO}_{3} \\
\mathrm{~cm}\end{array}$ & Temp. & $\begin{array}{c}\text { Strom- } \\
\text { stärke } \\
\text { Amp. }\end{array}$ & $\begin{array}{l}\text { Daner } \\
\text { Stdn. }\end{array}$ & $\begin{array}{c}\text { Nieder- } \\
\text { schlag } \\
\text { Besch. }\end{array}$ & $\begin{array}{c}\text { Diff. } \\
0\end{array}$ \\
\hline 0.0707 & 0.0707 & 0.8 & 50 & $0.1 ; 0.04$ & $1^{3 / 4}$ & fest, hell & 0.00 \\
\hline 0.0866 & 0.08155 & 0.4 & 50 & $0.1 ; 0.05$ & - & fest, hell & -0.12 \\
\hline 0.0994 & 0.0990 & 0.5 & 50 & 0.1 & $2^{3} / 4$ & fest & -0.4 \\
\hline 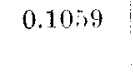 & 0.1056 & 1 & 50 & 0.05 & 3 & $\begin{array}{c}\text { hell mit } \\
\text { dunklen Rand }\end{array}$ & -0.3 \\
\hline 0.1059 & 0.1057 & 1 & ก0 & $0.1 ; \quad 0.05$ & 4 & hell, fest & -0.19 \\
\hline 0.1099 & 0.1096 & 0.6 & 50 & $0.1 ; 0.05$ & $2^{3} / 4$ & hell, fest & -0.28 \\
\hline 0.1099 & 0.1098 & 0.6 & 50 & $0.15 ; 0.05$ & $3^{1} / 2$ & - & -0.1 \\
\hline 0.1099 & 0.1098 & 0.6 & 50 & $0.1 ; \quad 0.05$ & 2 & dunkel & -0.1 \\
\hline 0.1348 & 0.1343 & 1 & 50 & $0.1 ; 0.05$ & $2^{1 / 2}$ & hell, fest & -0.3 \\
\hline 0.1596 & 0.1598 & 2 & 50 & $0.15 ; 005$ & $2^{3 / 4}$ & fest, hell & +0.12 \\
\hline 0.1667 & 0.1663 & 2 & 50 & $0.15 ; 0.05$ & $2^{3 / 4}$ & fest, hell & -0.25 \\
\hline 0.1774 & 0.1769 & 0.8 & 50 & $0.15 ; 0.05$ & 3 & dunkel & -0.28 \\
\hline 0.1774 & 0.1770 & 0.8 & 50 & $0.15 ; 0.05$ & $3^{1 / 4}$ & dunkel & -0.23 \\
\hline 0.1774 & 0.1771 & 0.8 & 50 & $0.15 ; 0.05$ & 4 & fest & -0.16 \\
\hline 0.1774 & 0.1772 & 0.8 & 50 & $0.15 ; 0.05$ & 3 & fest & -0.11 \\
\hline 0.1774 & 0.1772 & 0.8 & 50 & $0.15 ; 0.05$ & $31 / 4$ & fest & -0.11 \\
\hline 0.1774 & 0.1773 & 0.8 & 50 & $0.15 ; 0.05$ & 3 & dunkel & -0.06 \\
\hline 0.1988 & 0.1990 & 1 & 50 & $0.15 ; 0.05$ & 4 & fest, fleckig & +0.1 \\
\hline 0.21 .66 & 0.2162 & 1 & 50 & $0.15 ; 0.05$ & $21^{1} / 2$ & - & -0.19 \\
\hline 0.2198 & 0.2196 & 1 & 50 & $0.15 ; 0.05$ & 4 & fest & -0.09 \\
\hline 0.4266 & 0.4265 & 4 & 50 & $0.02 ; 0.05$ & - & fest & -0.62 \\
\hline
\end{tabular}

NB. Die in der fünften Kolumne angeführten Zahlen vor dem Semikolon bedeuten die anfängliche Stromstärke, die hinter dem Semikolon die Stromstärke, bei welcher die Elektrolyse zu Ende geführt wurde.

Kälte. Erst nach längerem Stehen beginnt die Lösung sich zu zersetzen; desgleichen zersetzt sie sich beim Erwärmen.

Die Eigenschaft des Glycerins, verhältnismälsig viel Wismutnitrat zu lösen, giebt uns ein Mittel an die Hand, eine Wismutlösung herzustellen, welche das Lösungsmittel nicht im Überschufs enthält und den Zusatz freier Säuren vermeidet.

Der auf diese Weise erhaltene Metallniederschlag ist hell und fest, jedoch ist Bewegung des Elektrolyten erforderlich.

Die Dauer der Elektrolyse ist etwa dieselbe wie die bei Anwendung salpetersaurer Lösungen.

Die günstigste Temperatur ist $50^{\circ}$. An der Anode tritt Super- 
oxyd in geringer Menge auf, das jedoch bis zur Beendigung der Elektrolyse stets wieder verschwindet.

Die Analyse wurde in folgender Weise ausgeführt:

In $2-4 \mathrm{ccm}$ einer Glycerinlösung, welche aus zwei Teilen Wasser und einem Teil käuflichem Glycerin bestand, löste ich 0.1 bis $0.3 \mathrm{~g}$ Wismutnitrat, verdünnte auf $150 \mathrm{ccm}$ und setzte die Liosung im Classenapparat einem Strome von $0.1 \mathrm{Amp}$. aus. Die Spannung betrug 2 Volt und die Temperatur wurde allmählich bis auf $50^{\circ}$ gesteigert. Ebenso wie aus der Fällung bei salpetersaurer Lösung mufs die Stromstärke nach etwa einer Stunde auf $0.05 \mathrm{Amp}$. verringert werden, da sonst der Niederschlag gegen Ende der Analyse schwammig ausfällt.

Bei Verwendung einer innen mattierten Kathodenschale erhält man hellere und dichtere Niederschläge, als in einer glatten Schale.

Versuche mit Lösungen von $\mathrm{Bi}\left(\mathrm{NO}_{3}\right)_{3} \cdot 5 \mathrm{H}_{2} \mathrm{O}$ in verdünntem Glycerin bei bewegtem Elektrolyten.

\begin{tabular}{c|c|c|c|c|c|c|c|c}
\hline $\begin{array}{c}\text { An- } \\
\text { gewandt }\end{array}$ & $\begin{array}{c}\text { Ge } \\
\text { funden } \\
g\end{array}$ & $\begin{array}{c}\text { Glycerin } \\
\text { ccm }\end{array}$ & Temp. & $\begin{array}{c}\text { Strom- } \\
\text { stärke } \\
\text { Amp. }\end{array}$ & $\begin{array}{c}\text { Dauer } \\
\text { Stdn. }\end{array}$ & $\begin{array}{c}\text { Nieder- } \\
\text { schlag } \\
\text { Besch. }\end{array}$ & $\begin{array}{c}\text { Diff. } \\
\text { I } \\
\%\end{array}$ & $\begin{array}{c}\text { Diff. } \\
\text { II } \\
0 / 0\end{array}$ \\
\hline 0.0683 & 0.0595 & 2.5 & 45 & $0.07 ; 0.05$ & $31 / 4$ & dunkel & +0.23 & +0.10 \\
0.1277 & 0.1278 & 4 & 60 & 0.05 & $4 y^{1 / 2}$ & fest, hell & +0.07 & +0.03 \\
0.1305 & 0.1302 & 3 & 65 & $0.1 ; 0.07$ & $2 / / 2$ & fest & -0.23 & -0.10 \\
0.1557 & 0.1561 & 4 & 60 & $0.1 ; 0.05$ & $2^{1 / 4}$ & fest & +0.24 & +0.10 \\
0.1516 & 0.1517 & 4 & 50 & $0.1 ; 0.05$ & 3 & fest, hell & +0.06 & +0.025 \\
0.1659 & 0.1660 & 4 & 70 & $0.1 ; 0.05$ & - & fest & +0.06 & +0.025 \\
0.3491 & 0.3482 & 12 & 60 & $0.25 ; 0.05$ & $2^{1 / 2}$ & fest & -0.26 & -0.11 \\
0.3657 & 0.3647 & 4 & 60 & $0.15 ; 0.05$ & $3^{3 / 4}$ & fest & -0.28 & -0.12
\end{tabular}

NB. Die unter Diff. I angeführten Prozentzahlen sind auf das Gewicht des berechneten Bi bezogen, die unter Diff. II auf das Gewicht des thatsächlich angewandten $\mathrm{Bi}\left(\mathrm{NO}_{3}\right)_{3} .5 \mathrm{H}_{2} \mathrm{O}$. Die Zahlen in der dritten Kolumne bedeuten die zur Lösung verwendeten Mengen der oben angegebenen Lösung vou 1 Teil Glyecrin und 2 Teilen Wasser.

In den beiden von mir ausgearbeiteten Bestimmungsweisen des Wismuts liegen zwei elektrolytische Methoden vor, welche den am Anfang dieser Arbeit aufgeführten Grundsätzen für quantitative elektrolytische Bestimmungen entsprechen, während dies bei den in Betracht gezogenen äiteren Methoden stets in Bezug auf einen oder 
mehrere Punkte nicht der Fall ist, sei es, dals, wie bei den Arbeiten von Thomas, Smith und Knerr, die Herstellung der Lösung Schwierigkeiten bietet oder die zur Anwendung kommenden Mengen allzu gering sind, sei es, dafs die allzulange Dauer der Analyse ihre praktische Ausführung erschwert, wie dies bei der Classen-EuiasBERG'schen Methode der Fall ist, oder dals die Beschaffenheit des erhaltenen Niederschlages zu wünschen übrig lälst, was für fast sämtliche Methoden gilt, falls ein ganz geringes Mafs der angewendeten Mengen überschritten wird.

Von diesen Fehlern sind die beiden von mir vorgeschlagenen Methoden frei, wenn die angegebenen Versuchsbedingungen genau eingehalten werden. Die Ausgangsmaterialien, Wismutoxyd und Wismutnitrat, können in völlig ausreichender Reinheit erlialten werden und sind in den Lösungsmitteln, Salpetersäure bezw. verdünntem Glycerin, leicht löslich.

Die Dauer geht nicht über die durchschnittliche, zur Abscheidung der üblichen Mengen eines Metalls nötige Zeit hinaus und der Metallniederschlag wird in derart fester Form erhalten, dals er ohne Verlust ausgewaschen werden kann.

Die Mengen, in welchen ich das Wismut zur Anwendung brachte, bleiben hinter den bei der Elektrolyse anderer Metallsalze gewöhnlich angewandten Mengen nicht zurück.

Die Elektrolysen wurden im Classenapparat ausgeführt unter Benutzung eines Rührwerks. In Bezug auf das letztere könnte geltend gemacht werden, dals das Rührwerk nicht unbedingt zu den allgemein üblichen Hilfsmitteln bei der Elektrolyse gezählt werden könne. Dieser Einwurf ist insofern gerechtfertigt, als allerdings das Rührwerk noch nicht in dem Mafse bei der Elektrolyse benutzt wird, als der Bedeutung der Sache entspricht. Während es bei sonstigen, insbesondere technischen elektrolytischen Arbeiten weitgehende Verwendung findet, ist es bei den quantitativen Bestimmungen der Metalle sozusagen noch gar nicht eingeführt. Von welcher Wichtigkeit es aber auch für analytische Arbeiten ist, beweist die vorliegende Arbeit, aus welcher hervorgeht, dals das Rühren während der Elektrolyse der Wismutsalze nicht nur bezüglich der Dauer und der Beschaffenheit des Metallniederschlages günstig wirkt, was ja auch bei vielen anderen Metallen der Fall ist, sondern geradezu als unerläfsliche Bedingung für die Ausführung der Ellektrolyse anzusehen ist. 
Die vorliegende Arbeit wurde im Wintersemester 1897/98 im technologischen Institut der Universität Würzburg auf Anregung des Herrn Professor Medrous begonnen, während des Sommersemesters 1898 daselbst und des Wintersemesters 1898/99 im elektrochemischen Institut des Herrn Professor Dumfenanbach an der technischen Hochschule in Darmstadt fortgesetzt und im Sommersemester 1899 dort beendigt.

Technolog. Institut der Universität Wïrzburg.

Bei der Redaktion eingegangen am 11. Januar 1901. 